\title{
The year 2013 in the European Heart Journal - Cardiovascular Imaging. Part I
}

\author{
Thor Edvardsen 1,2,3, Sven Plein ${ }^{4}$, Antti Saraste ${ }^{5}$, Luc A. Pierard ${ }^{6}$, Juhani Knuuti ${ }^{5}$, \\ Gerald Maurer ${ }^{7}$, and Patrizio Lancellotti ${ }^{6 *}$
} ${ }^{1}$ Department of Cardiology, Oslo University Hospital, Rikshospitalet, Oslo, Norway; ${ }^{2}$ Faculty of Medicine, University of Oslo, Oslo, Norway; ${ }^{3}$ Centre of Cardiological Innovation, Oslo
University Hospital, Rikshospitalet, Oslo, Norway; ${ }^{4}$ University of Leeds, Leeds, UK; ${ }^{5}$ Turku PET Centre and Heart Center, Turku University Hospital and University of Turku, Turku,
Finland; ${ }^{6}$ GIGA Cardiovascular Sciences, Heart Valve Clinic, Imaging Cardiology, University of Liège Hospital, CHU Sart Tilman, Liège, Belgium; and ${ }^{7}$ Division of Cardiology, Department of
Internal Medicine II, Medical University of Vienna, Vienna, Austria

Received 19 April 2014; accepted after revision 22 April 2014

The new multimodality cardiovascular imaging journal, European Heart Journal - Cardiovascular Imaging, was created in 2012. Here, we summarize the most important studies from the journal's second year in two articles. Part I of the review will focus on studies in myocardial function, myocardial ischaemia, and emerging techniques in cardiovascular imaging, and Part II will focus on valvular heart diseases, heart failure, cardiomyopathies, and congenital heart diseases.

Keywords multimodality cardiovascular imaging $\bullet$ echocardiography $\bullet$ nuclear $\bullet$ CMR $\bullet$ CT

\section{Introduction}

The multimodality European Heart Journal - Cardiovascular Imaging has successfully transitioned from an exclusive echocardiographic journal 2 years ago. The journal now serves as an important resource for general cardiologists, specialists in all imaging modalities, and other physicians working in the field of cardiovascular imaging. In two articles, we highlight the most important studies that were published in the journal in 2013. Part l is focused on studies in myocardial function, myocardial ischaemia, and emerging techniques in cardiovascular imaging.

\section{Recommendations from the European Association of Cardiovascular Imaging}

In addition to publishing original scientific studies, another important assignment for European Heart Journal - Cardiovascular Imaging is to publish position papers, recommendations and expert consensus papers from the European Association of Cardiovascular Imaging (EACVI). In 2013, the EACVI published three recommendations on echocardiographic topics ${ }^{1-3}$ and one expert consensus in the field of multimodality imaging. ${ }^{4}$

\section{Myocardial function}

During the last few years, it has been apparent that assessment of myocardial deformation by speckle-tracking echocardiography adds important information to functional assessment by ejection fraction (EF) only. ${ }^{5-7}$ Knowledge of myocardial function is pivotal in the diagnosis and risk stratification of patients with decreased myocardial function. ${ }^{8}$ The most powerful method is left ventricular (LV) global longitudinal strain (GLS) by two-dimensional (2D) speckletracking strain. This was further confirmed in a prospective study of 425 patients referred for cardiac surgery. ${ }^{9}$ This study demonstrated that GLS was an independent predictor, superior to EF, for early postoperative mortality after adjustment to EuroSCORE.

In an era of multimodality imaging, it is important to know how values compare between imaging modalities. A study by Puntmann et al. ${ }^{10}$ compared cardiovascular magnetic resonance (CMR) with transthoracic echocardiography (TTE) derived measurements of LV chamber dimensions and wall thickness. In 101 subjects, including 33 patients with dilated LV, good agreement between CMR and TTE, with a three-chamber CMR approach agreeing best with TTE.

Knowledge of myocardial function in cancer survivors after anthracycline treatment is important. A study from Yu et al. ${ }^{11}$ showed that children had impaired subendocardial circumferential deformation and apical rotation with consequential reduction of transmural circumferential strain and rotation gradients.

Current strain technology by different vendors is not compliant and different absolute strain values have been reported when comparing equipment from two or more vendors. It seems, however, that longitudinal strain is very reproducible, while other deformation directions show poorer reproducibility data. Therefore, the EACVI initiated a standardized approach to deformation imaging jointly

*Corresponding author. Tel: +3243667194; Fax: +3243667195, Email: plancellotti@chu.ulg.ac.be.

Published on behalf of the European Society of Cardiology. All rights reserved. (C) The Author 2014. For permissions please email: journals.permissions@oup.com. 
with the American Society of Echocardiography (ASE). ${ }^{12}$ The leaders of the EACVI and the ASE invited representatives from all vendors to participate in a concerted effort to reduce intervendor variability of strain measurement. The experience of the committee so far has demonstrated the potential for co-operation among vendors and the imaging societies. The first report will be published during 2014 in our journal. A recent comparison between 3D strain from two different vendors showed that the agreement was poor except for longitudinal strain assessment ${ }^{13}$ and confirmed the need for a standardization process.

Echocardiographic machines and software are constantly improving in regard to image quality, spatial resolution, and more. These improvements might lead to slightly different measurements. It will therefore be important to check normal ranges with newer equipment and software. The aim of the Normal Reference Ranges for Echocardiography Study (NORRE Study) is to obtain a set of 'normal values' for cardiac chamber geometry and function in a large cohort of healthy Caucasian individuals (25-75 years) using both conventional and advanced echocardiographic techniques. ${ }^{14}$ The first results were published in the journal in the beginning of 2014. ${ }^{15}$ The NORRE Study was performed in 22 laboratories accredited by the EACVI and in one laboratory in the USA accredited by Intersocietal Commission for the Accreditation of Echocardiography Laboratories. The final sample size was 734 normal subjects, in whom M-mode, 2D, and 3D imaging, colour Doppler, pulsed-wave Doppler, pulsed-wave tissue Doppler, and colour tissue Doppler imaging data were assessed. All studies were analysed in a central echocardiographic core laboratory for quantitative analysis. The first article presented a comprehensive analysis of chamber quantification in the large NORRE cohort. Another study provides normative values for right atrial volumes and function measured by $3 D$ and 2D speckle-tracking echocardiography. ${ }^{16}$ Assessment of left atrial (LA) mechanical function using speckle-tracking echocardiography was found to be a valid approach and clinically feasible compared with transoesophageal echocardiography. ${ }^{17}$

Assessment and interpretation of diastolic function remain difficult. The introduction of $e^{\prime}$ was launched as a solution to many of these problems, but controversies about its value persist. The value of $e^{\prime} / a^{\prime}$ is not yet determined, but an important contribution to a better knowledge on how to use this ratio was proposed by Kim et al. ${ }^{18}$ They studied determinants of preserved diastolic function at the lateral annulus in in 1166 consecutive patients with isolated diastolic dysfunction. They found that patients with lateral $e^{\prime} / a^{\prime}>1$ were younger, male, and had a lower prevalence of diabetes and hypertension and showed less evidence of LV diastolic dysfunction and structural remodelling as LV hypertrophy and LA enlargement, than those with a lateral $e^{\prime} / a^{\prime}<1$. It is also controversial whether septal or lateral $e^{\prime}$ should be the preferred measurement. Galderisi et al. ${ }^{19}$ showed that the lateral $e^{\prime}$ was the most accurate parameter to predict increased LV filling pressure in patients with coronary artery disease (CAD).

The complicated structure of the right ventricle (RV) makes it difficult to image with echocardiography. It consists of an inflow part including the tricuspid valve apparatus; a trabecular part that includes pronounced trabeculations; and an outflow tract consisting of a muscular infundibulum, separating the tricuspid from the pulmonary valve. ${ }^{20}$
The evaluation of the RV function has a very significant role as a prognostic factor in patients with myocardial infarction (MI). Threedimensional echocardiography may offer benefits for RV assessment than 2D echocardiography. In a recent study of 85 patients with RV infarct and also with inferior myocardial infarct, ${ }^{21}$ the use of RV $s^{\prime}$ as a parameter of RV performance was of similar value to discover $\mathrm{RV} \mathrm{Ml}$ as the use of 3D echocardiography.

Knowledge of RV and atrial function is also important in pulmonary hypertension. In a porcine model of RV chronic pressure overload, Guihaire et al. ${ }^{22}$ found that non-invasive indices of RV function are markers of ventricular-arterial coupling rather than ventricular contractility. The widely used RV fractional area change, tricuspid annular plane systolic excursion, peak systolic tricuspid annular velocity $(s)^{\prime}$, and the RV myocardial performance index were all found to better correlate to ventricular-arterial coupling as assessed by RV endsystolic elastance/pulmonary arterial elastance.

\section{Ischaemic heart disease}

The mortality from atherosclerotic cardiovascular diseases (CVDs) is steadily decreasing in many countries in Western Europe. CVD remains, however, the major cause of death worldwide. ${ }^{23}$ This patient group is also the largest group undergoing cardiac imaging procedures in Europe. Traditional risk scores have many limitations and are less accurate in subgroups as diabetics, women, younger patients, and elderly subjects. The role of cardiovascular imaging in detecting subclinical atherosclerosis is increasing and detection of de facto initial disease might overcome some limitations of conventional risk stratification.

CMR has an expanding range of indications and clinical applications. $^{24}$ It is particularly useful for in vivo imaging of $\mathrm{Ml}$ with methods available for delineation of myocardial oedema, necrosis/ fibrosis, and ischaemia. In an ex vivo pig model of acute MI, Ubachs et al. validated $T_{2}$-weighted CMR for the quantification of myocardium at risk against single-photon emission computed tomography (SPECT) and infarct size against a histological reference standard. The study showed that CMR accurately determined area at risk, infarct size, and the derived measure of myocardial salvage. ${ }^{25}$

Several studies published in 2013 confirmed the prognostic value of CMR in risk stratification after acute MI. In 309 patients, 3-month infarct size $\geq$ median by late gadolinium enhancement (LGE) CMR was a strong and independent predictor of outcome with an adjusted hazard ratio of 1.13 per $1 \%$ increase. $^{26}$ In a second study of 199 patients with acute ST elevation (STE) MI, CMR measures on LA function were independently associated with the outcome. ${ }^{27}$

Other studies highlighted the value of combined low-dose dobutamine and LGE CMR in the prediction of remodelling, ${ }^{28}$ which was also the topic of a meta-analysis, ${ }^{29}$ and the infarct border zone by LGE in the prediction of hard clinical endpoints. ${ }^{30}$ Other CMR outcome measures were reviewed in the European Heart Journal Cardiovascular Imaging by Mavrogeni et al. ${ }^{31}$

Assessment and quantification of myocardial blood flow (MBF) by CMR is increasingly used in clinical practice and now enshrined in European guidelines. Ebersberger et al. evaluated perfusion CMR at $3 \mathrm{~T}$ in 116 patients with suspected or known CAD against the invasive reference standard of pressure-wire-derived fractional flow reserve (FFR). CMR had a sensitivity, specificity, and positive and negative 
predictive values at the patient level of $85,87,77$, and $92 \%$, respectively. ${ }^{32}$ In an accompanying editorial, Plein and Motwani discussed the appropriateness of using FFR as an endpoint for non-invasive imaging studies, pointing out that FFR was initially validated against noninvasive imaging. The authors propose that validated imaging tests such as positron emission tomography may be more appropriate endpoints for such studies. ${ }^{33}$

The use of regional GLS by echocardiography in patients with $\mathrm{Ml}$ has been extensively studied. The superiority of these parameters to wall motion score index and EF in patients with acute STE MI and relatively preserved LV function was recently demonstrated by Cimino et al. ${ }^{34}$

Recent echocardiographic machines have the ability to assess deformation parameters from the different myocardial layers. However, the techniques differ between vendors and their clinical value is not established. A study comparing strain-encoded (SENC) CMR and 2D speckle-tracking echocardiography demonstrates that both methods can discriminate between different transmurality categories of $\mathrm{Ml}$ with similar accuracy. ${ }^{35}$ An experimental study could not show superiority of multilayer analyses in the assessment of reperfusion. ${ }^{36}$

Coronary flow reserve (CFR) assessment by transthoracic ultrasound of the left anterior descending (LAD) artery during dipyridamole stress echocardiography has extensively been studied. In a further study, CFR could predict adverse events when added to standard evaluation in patients with acute coronary syndrome (ACS) even in the absence of LAD disease. ${ }^{37}$

Intravascular ultrasound (IVUS) is not routinely in use in most of the patient groups, but has an important role in research. Culprit lesion-positive arterial remodelling by IVUS was associated with a poor long-term prognosis in patients with $\mathrm{ACS}^{38}$ This technique may be a clinically useful marker of vulnerable patients.

The use of mitral annular plane systolic excursion measurement is still helpful to evaluate LV systolic function in the case of poor sonographic windows. ${ }^{39}$

It has been commonly believed that reduced myocardial flow reserve (MFR) as a sign of early coronary dysfunction is linked with the subsequent development of CAD. In a study by Uusitalo et al., ${ }^{40}$ coronary calcium score (CCS) was measured 11 years after measurement of CFR in 77 healthy, lean, normotensive, non-smoking, and non-diabetic men (mean age 35 years). Thirty-nine percent of subjects had a CCS $>0$, but none had clinical symptoms or evidence of ischaemia in stress echocardiography. At baseline, the average CFR was comparable in individuals with or without coronary calcium in the follow-up study, suggesting that coronary reactivity does not predict the presence of coronary calcification in asymptomatic men with very low likelihood of CAD at least with 11 years of follow-up.

Farhad et al. ${ }^{41}$ investigated the added value of the quantitative MBF and the MFR in predicting major adverse cardiovascular events in patients with suspected myocardial ischaemia. MBF was quantitated using ${ }^{82} \mathrm{Rb}$ and positron emission tomography in 351 patients. During a median follow-up of 624 days, 35 major adverse cardiac events (MACEs) occurred. As expected, MACE rate was higher in patients with ischaemia than those without, but the quantitative MBF had independent prognostic power in addition to standard analysis. The authors concluded that MBF quantification has an added value allowing further risk stratification in patients with normal and abnormal perfusion images.
Adenosine stress CT myocardial perfusion imaging could enable the detection of haemodynamic significance of intermediate coronary stenosis detected by CT coronary angiography (CTCA). An experimental study demonstrated that dynamic dual source CT can quantitatively measure MBF and identify regional reductions of MBF during adenosine stress over a wide range of flow-limiting coronary artery obstruction severities with a good correlation to coronary blood flow obtained with intracoronary flow probe or FFR measurements. ${ }^{42}$

The combined or hybrid imaging of myocardial perfusion is gaining increasing interest and various combinations (CT + SPECT, CT + $P E T$, and $C T+C M R$ ) have been investigated. In a study by Schaap et al., ${ }^{43}$ hybrid SPECT and CT coronary angiography were evaluated in 98 patients and the results of the hybrid approach were compared with standalone SPECT and CTCA for the diagnosis of significant CAD. Parallel to what has been shown earlier, the sensitivities of SPECT and CT angiography were high (93 and 98\%), but the specificities were lower (79 and 62\%). Hybrid analysis of SPECT and CT angiography improved the overall performance but especially the specificity (95\%) without losing sensitivity (96\%). The authors confirmed that the combination of anatomy and function improves the performance of the diagnostic test.

\section{Cardiac CT and CAD}

CTCA is a useful tool for the detection and especially ruling out obstructive CAD in selected patients with stable angina pectoris. In a retrospective cohort of 498 stable, symptomatic patients with a lowintermediate probability of CAD referred for either CTCA or exercise-stress test, a frontline diagnostic strategy using $C T$ incurred lower costs related to downstream diagnostic utilization, ambulatory visits, and cardiovascular medication than exercise-stress test-based strategy. ${ }^{44}$ Meta-analyses of randomized controlled trials showed that CTCA can be effective and safe as the first imaging test to exclude significant CAD also in low-to-intermediate risk patients with acute chest pain in the emergency department. However, downstream utilization of resources is still a matter of debate since different results in the use of subsequent invasive coronary angiographies and revascularizations have been detected. ${ }^{45,46}$

Evaluation of coronary stents by CTCA remains challenging because partial volume or blooming artefacts from the highly attenuated stent struts cause problems for the delineation of the coronary stent lumen. The study by Gebhard et al. ${ }^{47}$ suggested that adaptive statistical iterative reconstruction may be useful in CTCA evaluation of these patients by improving intra-stent luminal area, diameter visualization, and image quality compared with the standard filtered back projection reconstruction.

Myocardial bridging, i.e. intramyocardial course of a coronary artery causing it to be covered by a bridge of myocardium, can be detected by CTCA. Rubishtein et al. ${ }^{48}$ found a relatively high (35\%) prevalence of myocardial bridging among a cohort of 334 symptomatic patients referred for CCTA and no obstructive CAD. However, there was no association between myocardial bridging and increased risk for cardiovascular death or MI during 6 years of follow-up.

Cardiac CT has potential to improve cardiovascular risk assessment over conventional risk factors of CAD by the detection of coronary calcium and non-calcified plaque as reviewed by Mureddu 
et al. ${ }^{23}$ Obesity is associated with the presence of CAD, but the relationships between body mass index (BMI) and conventional risk factors that often co-exist with obesity are complex. In a multi-ethnic, asymptomatic population of 1212 young men, obesity was associated with the presence of coronary artery calcium independently of the conventional risk factors of CAD. ${ }^{49}$ Similarly, among 13874 patients without known CAD undergoing CTCA, individuals with increased BMI had greater prevalence, extent, and severity of CAD that was not fully explained by the presence of traditional risk factors. ${ }^{50}$ Furthermore, a higher BMI was independently associated with increased risk of MI during an average of 2.4-year follow-up. In a cohort of 1535 individuals without clinically manifested CAD, the presence of chronic obstructive pulmonary disease in long-term smokers was associated with that of subclinical CAD measured by CCS independently of traditional cardiovascular risk factors. ${ }^{51}$ Although CAD is one of the underlying mechanisms of atrial fibrillation, Den Uijl et al. ${ }^{52}$ found that in the CTCA prior to radiofrequency catheter ablation for atrial fibrillation, obstructive CAD or the presence of coronary atherosclerosis was not associated with a higher risk for atrial fibrillation recurrence after ablation.

Low-attenuation plaque has been widely investigated as a marker for atherosclerotic plaque vulnerability. Comparison with histology in explanted hearts showed that low-attenuation plaque in CTA can be used as a marker for lipid core plaque, i.e. plaque with a large lipid/necrotic core. ${ }^{53}$ However, the (semi)-automated plaque assessment tools based on measurement of the area between the inner and outer vessel walls are prone to errors if cross-sections without any visually apparent plaque are included in the image analysis.

\section{Recent imaging techniques}

\section{Pocket-size imaging devices}

The miniaturization and improvements in technology have led to the development of pocket-size imaging devices (PSIDs) with good image quality and excellent portability. PSIDs are now widely available, and their potential role in a hospital environment has been investigated but still remains undefined. ${ }^{54}$ One study demonstrated that PSID can provide a valuable alternative to TTE in the presence of focused clinical questions and can provide an efficient way of delivering a ward-based transthoracic echo service. Another study showed that the use of PSID after only brief bedside training greatly improved the clinical diagnosis of medical students and junior doctors, over and above history, physical examination, and ECG findings. ${ }^{55} \mathrm{~A}$ third study showed that PSID used by a trained cardiologist has good diagnostic accuracy in the emergency setting compared with a high-end echocardiograph. ${ }^{56}$

\section{Contrast echocardiography}

Myocardial contrast echocardiography has many advantages and allows better assessment of wall motion, LV opacification and in many patients myocardial perfusion. ${ }^{57}$ One major disadvantage is the price of the contrast agents. The use of contrast agents in the UK is, however, limited to $<4 \%$ of all transthoracic echocardiographic studies. ${ }^{58}$ Major barriers to the implementation of contrast use are the absence of cardiac imaging specialists directly supervising echocardiography departments and the training of sonographers to independently administer contrast. A new area for contrast was
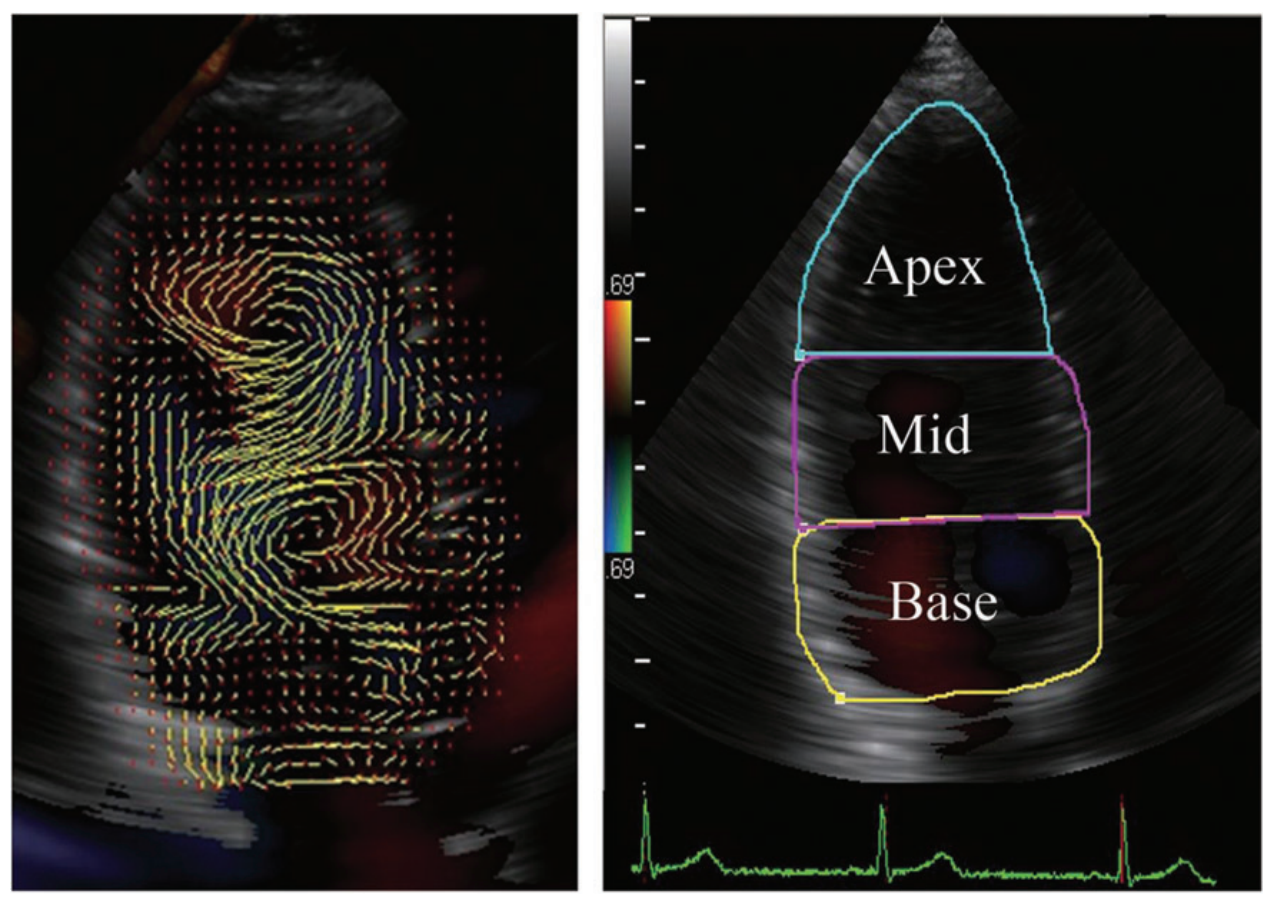

Figure I: Images of VFM. Velocity vectors were identified without angle dependency (left panel). The left ventricle was divided into three segments: base, mid, and apex (right panel). ${ }^{59}$ 
demonstrated by Rutz et al. They showed that contrast could assist in detecting chronic heart transplant rejection, i.e. cardiac allograft vasculopathy.

\section{New imaging techniques}

There is growing interest for intracardiac flow visualization. Recently developed vector flow mapping (VFM) enables evaluation of local flow dynamics without angle dependency. ${ }^{59}$

Retarded apical kinetic energy fluid dynamics could be detected using VFM and were shown to be closely associated with LV spherical remodelling in patients with high LA pressure (Figure 1).

Optical coherence tomography (OCT) is a recently developed intravascular imaging modality that allows detailed assessment of atherosclerotic plaque morphology, coronary stents, and abnormal tissue reactions associated with stent implantation. Kawamori et al. ${ }^{60}$ found that most cases of stent malapposition with a short distance between the strut and vessel wall, thrombus, tissue prolapse, or minor stent edge dissection improved during the 8-month followup. Park et al. ${ }^{61}$ found that the presence of intra-stent thrombi was seen as often in patients with or without aspirin/clopidogrel resistance 195 days after implantation of a drug-eluting stent. A study of Habara et al. ${ }^{62}$ revealed that the OCT morphological characteristics of drug-eluting stent restenotic tissue varied depending on time after stent implantation. Heterogeneous appearance of intima, thin-cap fibroatheroma-like pattern image, and intra-intimal microvessels were increased from the early $(<1$ year) to the very late $(>3$ years) phase. Matsuo et al. ${ }^{63}$ found that circulating levels of malondialdehyde-modified low-density lipoprotein, which is an oxidized low-density lipoprotein, were associated with the presence of thin-cap fibroatheroma plaque morphology in OCT in patients with either stable angina pectoris or ACS.

\section{Conclusion}

This review has summarized the studies in myocardial function, myocardial ischaemia, and emerging techniques published in 2013 in the European Heart Journal - Cardiovascular Imaging. Part II of the review will focus on valvular heart diseases, heart failure, cardiomyopathies, and congenital heart diseases.

\section{Conflict of interest: none declared.}

\section{Funding}

T.E. is funded by the Norwegian Research Council.

\section{References}

1. Lancellotti P, Tribouilloy C, HagendorffA, Popescu BA, Edvardsen T, Pierard LA et al. Recommendations for the echocardiographic assessment of native valvular regurgitation: an executive summary from the European Association of Cardiovascular Imaging. Eur Heart J Cardiovasc Imaging 2013;14:611-44.

2. Neskovic AN, Hagendorff A, Lancellotti P, Guarracino F, Varga A, Cosyns B et al. Emergency echocardiography: the European Association of Cardiovascular Imaging recommendations. Eur Heart J Cardiovasc Imaging 2013;14:1-11.

3. Cosyns B, Garbi M, Separovic J, Pasquet A, Lancellotti P, Education Committee of the European Association of Cardiovascular Imaging Association (EACVI). Update of the echocardiography core syllabus of the European Association of Cardiovascular Imaging (EACVI). Eur Heart J Cardiovasc Imaging 2013;14:837-9.

4. Lancellotti P, Nkomo VT, Badano LP, Bergler-Klein J, Bogaert J, Davin L et al. Expert consensus for multi-modality imaging evaluation of cardiovascular complications of radiotherapy in adults: a report from the European Association of Cardiovascular
Imaging and the American Society of Echocardiography. Eur Heart J Cardiovasc Imaging 2013;14:721-40.

5. Kearney LG, Lu K, Ord M, Patel SK, Profitis K, Matalanis G et al. Global longitudinal strain is a strong independent predictor of all-cause mortality in patients with aortic stenosis. Eur Heart J Cardiovasc Imaging 2012;13:827-33.

6. Zhao Y, Henein MY, Mörner S, Gustavsson S, Holmgren A, LindqvistP. Residual compromised myocardial contractile reserve after valve replacement for aortic stenosis. Eur Heart J Cardiovasc Imaging 2012;13:353-60.

7. Galderisi M, Esposito R, Schiano-Lomoriello V, Santoro A, Ippolito R, Schiattarella P et al. Correlates of global area strain in native hypertensive patients: a threedimensional speckle-tracking echocardiography study. Eur Heart J Cardiovasc Imaging 2012;13:730-8.

8. Edvardsen T, Plein S, Saraste A, Knuuti J, Maurer G, Lancellotti P. Theyear 2012 in the European Heart Journal - Cardiovascular Imaging: Part I. Eur Heart J Cardiovasc Imaging 2013;14:509-14.

9. Ternacle J, Berry M, Alonso E, Kloeckner M, CouetilJ-P, RandéJ-LD et al. Incremental value of global longitudinal strain for predicting early outcome after cardiac surgery. Eur Heart J Cardiovasc Imaging 2013;14:77-84.

10. Puntmann VO, Gebker R, Duckett S, Mirelis ], Schnackenburg B, Graefe M et al. Left ventricular chamber dimensions and wall thickness by cardiovascular magnetic resonance: comparison with transthoracic echocardiography. Eur Heart J Cardiovasc Imaging 2013;14:240-6.

11. Yu W, Li S, Chan GCF, Ha S, Wong SJ, Cheung Y. Transmural strain and rotation gradient in survivors of childhood cancers. Eur Heart J Cardiovasc Imaging 2013;14 $175-82$.

12. Thomas JD, Badano LP. EACVI-ASE-industry initiative to standardize deformation imaging: a brief update from the co-chairs. Eur Heart J Cardiovasc Imaging 2013;14: 1039-40.

13. Badano LP, Cucchini U, Muraru D, Al Nono O, Sarais C, lliceto S. Use of three-dimensional speckle tracking to assess left ventricular myocardial mechanics: inter-vendor consistency and reproducibility of strain measurements. Eur Heart J Cardiovasc Imaging 2013;14:285-93.

14. Lancellotti P, Badano LP, Lang RM, Akhaladze N, Athanassopoulos GD, Barone D et al. Normal Reference Ranges for Echocardiography: rationale, study design, and methodology (NORRE Study). Eur Heart J Cardiovasc Imaging 2013;14:303-8.

15. Kou S, Caballero L, Dulgheru R, Voilliot D, De Sousa C, Kacharava G et al. Echocardiographic reference ranges for normal cardiac chamber size: results from the NORRE Study. Eur Heart J Cardiovasc Imaging 2014; doi:10.1093/ehjci/jet284.

16. Peluso D, Badano LP, Muraru D, Dal Bianco L, Cucchini U, Kocabay G et al. Right atrial size and function assessed with three-dimensional and speckle-tracking echocardiography in 200 healthy volunteers. Eur Heart J Cardiovasc Imaging 2013;14: $1106-14$.

17. Leong DP, Penhall A, Perry R, Shirazi M, Altman M, Chong D et al. Speckle-tracking strain of the left atrium: a transoesophageal echocardiographic validation study. Eur Heart J Cardiovasc Imaging 2013;14:898-905.

18. Kim S-A, Park W-J, Kim HJ, Jo S-H, Kim H-S, Han S-J et al. Determinants of preserved diastolic function at the lateral annulus in patients with isolated diastolic dysfunction. Eur Heart J Cardiovasc Imaging 2013;14:443-8.

19. Galderisi M, Rapacciuolo A, Esposito R, Versiero M, Schiano-Lomoriello V, Santoro Cet al. Site-dependency of the E/e' ratio in predicting invasive left ventricular filling pressure in patients with suspected or ascertained coronary artery disease. Eur Heart J Cardiovasc Imaging 2013;14:555-61.

20. Mertens L, Hunter K. Imaging right ventricular shape and remodelling. Eur Heart /Cardiovasc Imaging 2013;14:311-2.

21. Kidawa M, Chizyński K, Zielińska M, Kasprzak JD, Krzeminska-Pakula M. Real-time $3 \mathrm{D}$ echocardiography and tissue Doppler echocardiography in the assessment of right ventricle systolic function in patients with right ventricular myocardial infarction. Eur Heart J Cardiovasc Imaging 2013;14:1002-9.

22. Guihaire J, Haddad F, Boulate D, Decante B, Denault AY, Wu J et al. Non-invasive indices of right ventricular function are markers of ventricular-arterial coupling rather than ventricular contractility: insights from a porcine model of chronic pressure overload. Eur Heart J Cardiovasc Imaging 2013;14:1140-9.

23. Mureddu GF, Brandimarte F, Faggiano P, Rigo F, Nixdorff U. Between risk charts and imaging: how should we stratify cardiovascular risk in clinical practice? Eur Heart J Cardiovasc Imaging 2013;14:401-16.

24. Von Knobelsdorff-Brenkenhoff F, Bublak A, El-Mahmoud S, Wassmuth R, Opitz C Schulz-Menger J. Single-centre survey of the application of cardiovascular magnetic resonance in clinical routine. Eur Heart J Cardiovasc Imaging 2013;14:62-8.

25. Ubachs JFA, Engblom H, Koul S, Kanski M, Andersson P, van der Pals J et al. Myocardium at risk can be determined by ex vivo $\mathrm{T} 2$-weighted magnetic resonance imaging even in the presence of gadolinium: comparison to myocardial perfusion single photon emission computed tomography. Eur Heart J Cardiovasc Imaging 2013;14: 261-8.

26. Lønborg J, Vejlstrup N, Kelbæk H, Holmvang L, Jørgensen E, Helqvist S et al. Final infarct size measured by cardiovascular magnetic resonance in patients with ST 
elevation myocardial infarction predicts long-term clinical outcome: an observational study. Eur Heart J Cardiovasc Imaging 2013;14:387-95.

27. Lønborg JT, Engstrøm T, Møller JE, Ahtarovski KA, Kelbæk H, Holmvang L et al. Left atrial volume and function in patients following ST elevation myocardial infarction and the association with clinical outcome: a cardiovascular magnetic resonance study. Eur Heart J Cardiovasc Imaging 2013;14:118-27.

28. Scott AE, Semple SIK, Redpath TW, Hillis GS. Low-dose dobutamine adds incremental value to late gadolinium enhancement cardiac magnetic resonance in the prediction of adverse remodelling following acute myocardial infarction. Eur Heart J Cardiovasc Imaging 2013;14:906-13.

29. Romero J, Kahan J, Kelesidis I, Makani H, Wever-Pinzon O, Medina H et al. CMR imaging for the evaluation of myocardial stunning after acute myocardial infarction: a meta-analysis of prospective trials. Eur Heart] Cardiovasc Imaging 2013;14:1080-91.

30. Robbers LFHJ, Delewi R, Nijveldt R, Hirsch A, Beek AM, Kemme MJB et al. Myocardial infarct heterogeneity assessment by late gadolinium enhancement cardiovascular magnetic resonance imaging shows predictive value for ventricular arrhythmia development after acute myocardial infarction. Eur Heart J Cardiovasc Imaging 2013;14:1150-8.

31. Mavrogeni S, Petrou E, Kolovou G, Theodorakis G, lliodromitis E. Prediction of ventricular arrhythmias using cardiovascular magnetic resonance. Eur HeartJ Cardiovasc Imaging 2013;14:518-25.

32. Ebersberger U, Makowski MR, Schoepf UJ, Platz U, Schmidtler F, Rose J et al. Magnetic resonance myocardial perfusion imaging at 3.0 Tesla for the identification of myocardial ischaemia: comparison with coronary catheter angiography and fractional flow reserve measurements. Eur Heart J Cardiovasc Imaging 2013;14:1174-80.

33. Plein S, Motwani M. Fractional flow reserve is a useful reference standard for myocardial perfusion studies with limitations: reply. Eur Heart J Cardiovasc Imaging 2014; 15:474-5.

34. Cimino S, Canali E, Petronilli V, Cicogna F, De Luca L, Francone M et al. Global and regional longitudinal strain assessed by two-dimensional speckle tracking echocardiography identifies early myocardial dysfunction and transmural extent of myocardial scar in patients with acute ST elevation myocardial infarction and relatively preserved LV function. Eur Heart J Cardiovasc Imaging 2013;14:805-11.

35. Altiok E, Neizel M, Tiemann S, Krass V, Becker M, Zwicker Cet al. Layer-specific analysis of myocardial deformation for assessment of infarct transmurality: comparison of strain-encoded cardiovascular magnetic resonance with 2D speckle tracking echocardiography. Eur Heart/ Cardiovasc Imaging 2013;14:570-8.

36. Moen CA, Salminen P-R, Dahle GO, Hjertaas J], Grong K, Matre K. Multi-layer radial systolic strain vs. one-layer strain for confirming reperfusion from a significant nonocclusive coronary stenosis. Eur Heart J Cardiovasc Imaging 2013;14:24-37.

37. Ascione L, Carlomagno G, Sordelli C, lengo R, Monda V, Severino S et al. Dipyridamole coronary flow reserve stratifies prognosis in acute coronary syndrome patients without left anterior descending disease. Eur Heart J Cardiovasc Imaging 2013;14:858-64.

38. Okura H, Kataoka T, Matsushita N, Shimeno K, Yoshiyama M, Yoshikawa J et al. Culprit lesion remodelling and long-term prognosis in patients with acute coronary syndrome: an intravascular ultrasound study. Eur Heart J Cardiovasc Imaging 2013;14: $758-64$.

39. Hu K, Liu D, Herrmann S, Niemann M, Gaudron PD, Voelker W et al. Clinical implication of mitral annular plane systolic excursion for patients with cardiovascular disease. Eur Heart J Cardiovasc Imaging 2013;14:205-12.

40. Uusitalo V, Saraste A, Kajander S, Luotolahti M, Wendelin-Saarenhovi M, Sundell J et al. The association between coronary flow reserve and development of coronary calcifications: a follow-up study for 11 years in healthy young men. Eur Heart J Cardiovasc Imaging 2013;14:812-8.

41. Farhad H, Dunet V, Bachelard K, Allenbach G, Kaufmann PA, Prior JO. Added prognostic value of myocardial blood flow quantitation in rubidium-82 positron emission tomography imaging. Eur Heart J Cardiovasc Imaging 2013;14:1203-10.

42. Rossi A, Uitterdijk A, Dijkshoorn M, Klotz E, Dharampal A, van Straten Met al. Quantification of myocardial blood flow by adenosine-stress $C T$ perfusion imaging in pigs during various degrees of stenosis correlates well with coronary artery blood flow and fractional flow reserve. Eur Heart/ Cardiovasc Imaging 2013;14:331-8.

43. Schaap J, Kauling RM, Boekholdt SM, Nieman K, Meijboom WB, Post MC et al. Incremental diagnostic accuracy of hybrid SPECT/CT coronary angiography in a population with an intermediate to high pre-test likelihood of coronary artery disease. Eur Heart J Cardiovasc Imaging 2013;14:642-9.

44. Nielsen LH, Olsen J, Markenvard J, Jensen JM, Nørgaard BL. Effects on costs of frontline diagnostic evaluation in patients suspected of angina: coronary computed tomography angiography vs. conventional ischaemia testing. Eur Heart J Cardiovasc Imaging 2013;14:449-55.
45. D'Ascenzo F, Cerrato E, Biondi-Zoccai G, Omedè P, Sciuto F, Presutti DG et al. Coronary computed tomographic angiography for detection of coronary artery disease in patients presenting to the emergency department with chest pain: a meta-analysis of randomized clinical trials. Eur Heart / Cardiovasc Imaging 2013;14:782-9.

46. Hulten E, Pickett C, Bittencourt MS, Villines TC, Petrillo S, Di Carli MF et al. Meta-analysis of coronary CT angiography in the emergency department. Eur HeartJ Cardiovasc Imaging 2013;14:607.

47. Gebhard C, Fiechter M, Fuchs TA, Stehli J, Müller E, Stähli BE et al. Coronary artery stents: influence of adaptive statistical iterative reconstruction on image quality using 64-HDCT. Eur Heart J Cardiovasc Imaging 2013;14:969-77.

48. Rubinshtein R, Gaspar T, Lewis BS, Prasad A, Peled N, Halon DA. Long-term prognosis and outcome in patients with a chest pain syndrome and myocardial bridging: a 64-slice coronary computed tomography angiography study. Eur Heart J Cardiovasc Imaging 2013;14:579-85.

49. Fujiyoshi A, Sekikawa A, Shin C, Masaki K, David Curb J, Ohkubo T et al. A crosssectional association of obesity with coronary calcium among Japanese, Koreans, Japanese Americans, and US whites. Eur Heart J Cardiovasc Imaging 2013;14:921-7.

50. Labounty TM, Gomez MJ, Achenbach S, Al-Mallah M, Berman DS, Budoff MJ et al. Body mass index and the prevalence, severity, and risk of coronary artery disease: an international multicentre study of 13,874 patients. Eur Heart J Cardiovasc Imaging 2013;14:456-63.

51. Rasmussen T, Køber L, Pedersen JH, Dirksen A, Thomsen LH, Stender S et al. Relationship between chronic obstructive pulmonary disease and subclinical coronary artery disease in long-term smokers. Eur Heart J Cardiovasc Imaging 2013;14 1159-66.

52. Den Uijl DW, Boogers MJ, Compier M, Trines SA, Scholte AJHA, Zeppenfeld K et al. Impact of coronary atherosclerosis on the efficacy of radiofrequency catheter ablation for atrial fibrillation. Eur Heart J Cardiovasc Imaging 2013;14:247-52.

53. Schlett CL, Ferencik M, Celeng C, Maurovich-Horvat P, Scheffel H, Stolzmann Pet al. How to assess non-calcified plaque in $C T$ angiography: delineation methods affect diagnostic accuracy of low-attenuation plaque by $\mathrm{CT}$ for lipid-core plaque in histology. Eur Heart J Cardiovasc Imaging 2013;14:1099-105.

54. Gianstefani S, Catibog N, Whittaker AR, loannidis AG, Vecchio F, Wathen PT et al. Pocket-size imaging device: effectiveness for ward-based transthoracic studies. Eur Heart J Cardiovasc Imaging 2013;14:1132-9.

55. Panoulas VF, Daigeler A-L, Malaweera ASN, Lota AS, Baskaran D, Rahman S et al. Pocket-size hand-held cardiac ultrasound as an adjunct to clinical examination in the hands of medical students and junior doctors. Eur Heart J Cardiovasc Imaging 2013; 14:323-30.

56. Testuz A, Müller H, Keller P-F, Meyer P, Stampfli T, Sekoranja L et al. Diagnostic accuracy of pocket-size handheld echocardiographs used by cardiologists in the acute care setting. Eur Heart / Cardiovasc Imaging 2013;14:38-42.

57. Plein S, Knuuti J, Edvardsen T, Saraste A, Piérard LA, Maurer G et al. The year 2012 in the European Heart Journal - Cardiovascular Imaging. Part II. Eur Heart J Cardiovasc Imaging 2013;14:613-7.

58. Bhattacharyya S, Khattar R, Lloyd G, Senior R, British Society of Echocardiography. Implementation of echocardiographic contrast agents into clinical practice: a United Kingdom National Health Service Survey on behalf of the British Society of Echocardiography. Eur Heart J Cardiovasc Imaging 2013;14:550-4.

59. Nogami Y, Ishizu T, Atsumi A, Yamamoto M, Kawamura R, Seo Y et al. Abnormal early diastolic intraventricular flow 'kinetic energy index' assessed by vector flow mapping in patients with elevated filling pressure. Eur Heart J Cardiovasc Imaging 2013;14:253-60.

60. Kawamori H, Shite J, Shinke T, Otake H, Matsumoto D, Nakagawa M et al. Natural consequence of post-intervention stent malapposition, thrombus, tissue prolapse, and dissection assessed by optical coherence tomography at mid-term follow-up. Eur Heart J Cardiovasc Imaging 2013;14:865-75.

61. Park J, Shin D-H, Kim B-K, Her A-Y, Kim YH, Choi HH et al. Relationship between aspirin/clopidogrel resistance and intra-stent thrombi assessed by follow-up optical coherence tomography after drug-eluting stent implantation. Eur Heart] Cardiovasc Imaging 2013;14:1181-6.

62. Habara M, Terashima M, Nasu K, Kaneda H, Yokota D, Ito T et al. Morphological differences of tissue characteristics between early, late, and very late restenosis lesions after first generation drug-eluting stent implantation: an optical coherence tomography study. Eur Heart J Cardiovasc Imaging 2013;14:276-84.

63. Matsuo Y, Kubo T, Okumoto Y, Ishibashi K, Komukai K, Tanimoto T et al. Circulating malondialdehyde-modified low-density lipoprotein levels are associated with the presence of thin-cap fibroatheromas determined by optical coherence tomography in coronary artery disease. Eur Heart J Cardiovasc Imaging 2013;14:43-50. 